\title{
Chronic Cocaine Self-Administration in Rhesus Monkeys: Impact on Associative Learning, Cognitive Control, and Working Memory
}

\author{
Jessica N. Porter, ${ }^{1,2}$ Adam S. Olsen, ${ }^{3}$ Kate Gurnsey, ${ }^{4}$ Brian P. Dugan, ${ }^{4}$ Hank P. Jedema, ${ }^{4}$ and Charles W. Bradberry ${ }^{1,2,3,4,5}$ \\ ${ }^{1}$ Center for Neuroscience at the University of Pittsburgh and ${ }^{2}$ Center for the Neural Basis of Cognition, University of Pittsburgh and Carnegie Mellon \\ University, Pittsburgh, Pennsylvania 15213, ${ }^{3}$ Department of Neuroscience, University of Pittsburgh, Pittsburgh, Pennsylvania 15260, ${ }^{4}$ Department of \\ Psychiatry, University of Pittsburgh, Pittsburgh, Pennsylvania 15213, and ${ }^{5}$ Veterans Affairs Pittsburgh Health Services, Pittsburgh, Pennsylvania 15240
}

Cocaine users display a wide range of cognitive impairments. Because treatment outcome is dependent on baseline cognitive ability, it is clinically important to understand the underlying neurobiology of these deficits. Therefore, it is crucial to determine whether cocaine exposure by itself is an etiological factor and, if so, to determine the overall nature of cognitive deficits associated with cocaine use. This will help to guide therapeutic approaches that address cognitive components of cocaine use to improve treatment outcome. We used rhesus monkeys in a longitudinal study in which 14 animals were characterized before assignment to matched control $(n=6)$ and cocaine self-administration $(n=8)$ groups. Self-administration took place on 4 consecutive days/week over 9 months, with a maximum (and typical) daily cumulative intake of $3.0 \mathrm{mg} / \mathrm{kg}$. Weekly cognitive assessments (total of 36 ) were conducted after a $72 \mathrm{~h}$ drug-free period. We used a stimulus discrimination task with reversal to evaluate associative learning and the cognitive control/flexibility needed to adapt to changes in reward contingencies. After extended self-administration, initial accuracy on the stimulus discrimination indicated intact associative learning. However, animals were impaired at maintaining high levels of accuracy needed to reach criterion and initiate the reversal. Increasing the reward contrast between stimuli permitted evaluation of reversal performance and revealed striking deficits in the cocaine group. Impairments in visual working memory were also observed using a delayed match-to-sample task. These results suggest a combination of generalized, possibly attentional, impairments, along with a more specific cognitive control impairment implicating orbitofrontal cortex dysfunction.

\section{Introduction}

There is increasing interest in the nature and etiology of cognitive deficits associated with drug dependence (Rogers and Robbins, 2001), arising from the likelihood that decisions to continue use despite negative outcomes reflect impairments in higher cognitive processing. Clinically, this is important because of evidence that therapeutic outcome is related to cognitive abilities at treatment onset (Teichner et al., 2002; Patkar et al., 2004; Aharonovich et al., 2006). However, the extent to which those deficits result from drug use rather than preexisting traits is unclear, making controlled animal studies necessary (Rogers and Robbins, 2001). Such studies avoid the confounds of preexisting differences, poly-drug use, varying length and amounts of drug use, and lifestyle stressors unique to drug using populations. Determining whether there is a causal relationship between cocaine

Received 0ct. 15, 2010; revised Jan. 13, 2011; accepted Feb. 2, 2011.

This work was supported by the Veterans Affairs Medical Research Service and National Institutes of Health/ National Institute on Drug Abuse Grants DA 25636 and DA 10331. Excellent technical support was provided by Gustavo Gomez, Kathryn Schwienteck, and Jessica Nicolazzo.

The authors declare no competing financial interests.

Correspondence should be addressed to C. W. Bradberry, Department of Psychiatry, University of Pittsburgh, 3501 Fifth Avenue, Room 4078, Pittsburgh, PA 15261. E-mail: bradberrycw@upmc.edu.

DOI:10.1523/JNEUROSCI.5426-10.2011

Copyright $\odot 2011$ the authors $\quad 0270-6474 / 11 / 314926-09 \$ 15.00 / 0$ self-administration and specific cognitive deficits was the primary aim of this study.

In addition to etiology, the specificity of cognitive impairments associated with cocaine use are unclear. One domain consistently impaired is cognitive control/flexibility (Garavan and Hester, 2007), reflected by increased perseverative responding subsequent to the reversal of reward contingencies (Fillmore and Rush, 2006; Ersche et al., 2008). The inability to adapt to altered reward contingencies does not appear to result from impaired associative learning in users who understand task contingencies from verbal instructions (Ersche et al., 2008), although previous animal studies indicate impaired acquisition of an unfamiliar task in which stimulus discriminations had to be learned by trial and error (Liu et al., 2008). Impaired working memory has been reported (O'Malley et al., 1992; Hoff et al., 1996; Bechara and Martin, 2004; Kübler et al., 2005; VerdejoGarcia and Perez-Garcia, 2007), although the literature is surprisingly mixed (Bolla et al., 1999; Pace-Schott et al., 2008). We have examined the issue of whether specific cognitive domains are affected by cocaine self-administration through the use of tasks that evaluate associative learning, cognitive control/flexibility, and visual working memory. This study complements previous studies examining effects of cocaine on reversal learning (Jentsch et al., 2002; Schoenbaum et al., 2004) and working memory (George et al., 2008). 
In this study, rhesus monkeys were extensively characterized on cognitive tasks before initiating an extended period of cocaine self-administration. After initiating self-administration, cognitive assessments were conducted weekly in drug-free animals to evaluate the impact of cocaine exposure on distinct cognitive domains. We used a stimulus discrimination/reversal task to assess associative learning and cognitive control/flexibility. The reversal component requires orbitofrontal cortex (Dias et al., 1996; Fellows and Farah, 2003; Hornak et al., 2004; Izquierdo et al., 2004; Murray and Izquierdo, 2007). A delayed match-to-sample task was used to assess visual working memory. Delayed matchto-sample performance has been linked to the ventrolateral prefrontal cortex (Wilson et al., 1993; Elliott and Dolan, 1999). We hypothesized that chronic cocaine exposure would impair reversal learning and working memory, thus implicating orbitofrontal cortex and ventrolateral prefrontal cortex dysfunction. Using tasks associated with distinct cognitive domains allowed us to address the question of whether cocaine use has a selective impact on particular aspects of cognitive function.

\section{Materials and Methods}

\section{Subjects}

Young adult (43-57 months at beginning of study) male rhesus macaque monkeys $(n=14)$ with no previous nonclinical drug exposure or behavioral training were used for the present study. During acquisition, animals were initially trained to target for food reinforcement and then habituated to pole and collar handling and placement in a behavioral primate chair (Primate Products). Water regulation was then established, and subjects were taught to use a sipper tube attached to the chair for water reinforcement. Animals were water regulated $5 \mathrm{~d} /$ week and were supplemented (weekly average of $25 \mathrm{ml} \cdot \mathrm{kg}^{-1} \cdot \mathrm{d}^{-1}$ ) at the end of each day after training and testing. Animals received ad libitum access to water over the weekend. Water regulation started at least $24 \mathrm{~h}$ before cognitive testing. Initially all monkeys were pair housed in standard stainless steel primate cages. Over time, incompatibility emerged among some pairs. When attempts at repairing were unsuccessful, monkeys were subsequently singly housed to avoid conflict injuries. At the end of the experiment, nine animals continued to be pair housed, whereas three controls and two cocaine monkeys had to be individually housed.

\section{Surgery}

Before baseline cognitive assessments, all animals had a vascular access port placed midscapula from which a catheter extended subcutaneously to an internal jugular vein. The vascular access port allows percutaneous nonstressful access to vasculature for cocaine self-administration without the need for a protective jacket and with reduced risk of infection because nothing is external to the skin (Wojnicki et al., 1994).

\section{Self-administration}

Both groups self-administered (either water or cocaine) in the chambers used for cognitive testing. Animals self-administered by touching an abstract shape on the touch screen for the required number of touches. Once the response requirement was met, a green border around the white screen would appear, and either cocaine (cocaine group) was administered intravenously via the vascular access port or water (control group) via a sipper tube. Contextual cues were also associated with reinforcement for later determination of their impact on behavior. These cues, consisting of a specific auditory sequence of tones and distinct visual border to the screen, were present throughout the entire session except during the time of reward delivery. During the cocaine selfadministration sessions, animals were allowed to self-administer up to six infusions of cocaine. After the first week, animals typically administered all six infusions. The cocaine group began self-administering an average daily dose $0.6 \mathrm{mg} / \mathrm{kg}(0.1 \mathrm{mg} / \mathrm{kg}$ per infusion, six infusions) of cocaine daily under a fixed ratio-3 (FR-3) schedule and 5 min timeout. Response requirements were gradually increased to a terminal FR-30, a $10 \mathrm{~min}$ timeout interval, and $3.0 \mathrm{mg} / \mathrm{kg}$ cumulative session dose (six 0.5
Cognitive Assessment and Self-Administration Schedule

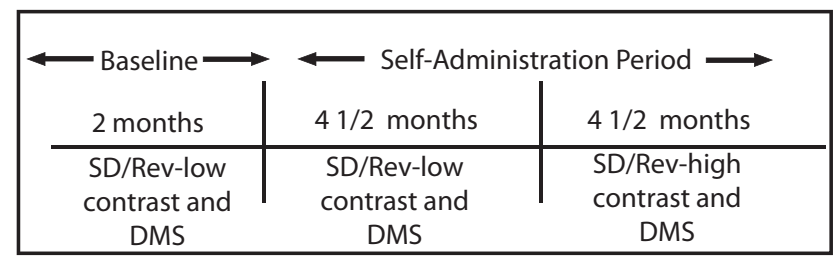

Figure 1. Timeline of self-administration and cognitive testing. Note the change in reward contrast for the stimulus discrimination (SD)/reversal task. DMS, Delayed match-to-sample.

\section{Table 1. Daily schedule for self-administration and cognitive assessment procedures}

\begin{tabular}{ll}
\hline Monday & Cognitive testing (SD/REV or DMS) \\
Tuesday & (1) Progressive ratio \\
& (2) Self-administration \\
Wednesday & Self-administration \\
Thursday & Self-administration \\
Friday & Self-administration \\
Saturday & No self-administration, ad libitum water \\
Sunday & No self-administration, water lines removed
\end{tabular}

Animals performed either the stimulus discrimination/reversal (SD/REV) or delayed match-to-sample (DMS) task every Monday and self-administered Tuesday through Friday. The progressive ratio evaluation of water reward efficacy on Tuesday took place in a morning session separate from the self-administration session in the afternoon. Animals had ad libitum water starting Friday after testing until water lines were removed on Sunday morning.

$\mathrm{mg} / \mathrm{kg}$ per infusions of $5 \mathrm{mg} / \mathrm{ml}$, delivered at a rate of $0.15 \mathrm{ml} / \mathrm{s}$ ). All animals reached the $3.0 \mathrm{mg} / \mathrm{kg}$ session dose over $15 \mathrm{~d}$ of gradually increasing the unit dose.

The control group animals began self-administering an average of 1.8 $\mathrm{ml} / \mathrm{kg}$ water under a FR-3 schedule (six infusions, $0.3 \mathrm{ml} / \mathrm{kg}$ per infusion). After $23 \mathrm{~d}$, the water amount was increased to $11.8 \mathrm{ml} / \mathrm{kg}$ water $(18$ infusions, $0.66 \mathrm{ml} / \mathrm{kg}$ per trial), and the schedule was switched to a random ratio of 5-15 with a timeout of 3.3 min to maintain cumulative amounts of lever pressing and chamber time similar to the cocaine group.

\section{Cognitive assessments}

All cognitive assessments took place in a sound attenuated chamber (model AB4240; Eckel Industries) fitted with a $40 \mathrm{~W}$ house light and white-noise generator. The E-prime software suite (Psychology Software Tools), coupled with a 15 inch touch screen (Elo Systems CarrollTouch), was used for all stimulus presentation, response recording, and data processing. Using baseline measures for each cognitive task described below, performance and age-matched control $(n=6)$ and experimental $(n=8)$ groups were established. Self-administration and cognitive assessments were organized as indicated in Figure 1 and Table 1. The animals self-administered Tuesday through Friday. Cognitive assessments were conducted on Mondays, when the animals had been drug free for $\sim 72 \mathrm{~h}$. A stimulus discrimination/reversal task was used to assess associative learning and cognitive control/flexibility, respectively, and a delayed match-to-sample task was used to assess visual working memory. Testing on the delayed match-to-sample task or the stimulus discrimination/reversal task alternated each week. On Tuesday mornings, a progressive ratio assessment of water reward efficacy took place, with self-administration beginning in a separate session Tuesday afternoon. Both groups self-administered and performed cognitive tasks contemporaneously.

\section{Stimulus discrimination/reversal task}

On each cognitive assessment day, three novel, visually distinct stimuli were used, thus requiring the subjects to learn a new operational stimulus-reward association at the beginning of each session. Each trial of the task began with presentation of a blue square that the animal had to touch, thereby indicating it was attending to the task. Touching the blue square led to presentation of two of the three stimuli, randomly presented left and right of midline. The stimuli were associated with a high, medium, or low water reward. On any given trial, an animal had a choice 
between a high or low, high or medium, or medium and low reward contingency. A correct response was recorded when the monkey touched the stimulus with the relatively greater reward. Once a criterion of 27 of 30 correct responses on consecutive trials were made, the contingency was reversed in that the high and low stimuli were switched, with the middle stimulus unchanged. Reaching the same performance criterion after the reversal resulted in presentation of a new set of three stimuli for a discrimination block. Animals were allowed to do as many discrimination and reversal trials as possible within a 200 trial session.

Two different reward levels were used to assess association learning (stimulus discrimination) and the cognitive flexibility needed to adapt to altered contingencies (reversal performance). The initial stimulus discrimination/reversal task had a low contrast in reward levels between stimuli (stimulus discrimination/reversal-low reward contrast). The stimuli were associated with a high $(0.1 \mathrm{ml} / \mathrm{kg})$, medium $(0.05 \mathrm{ml} / \mathrm{kg})$, or low $(0.02 \mathrm{ml} / \mathrm{kg})$ water reward. The stimulus discrimination/reversallow reward contrast was used to assess baseline performance (preadministration low contrast) across 10 daily sessions before initiating self-administration. The same reward values were used for nine sessions after initiation of self-administration (post-administration low contrast) over a period of 4-5 months. During the post-administration low contrast period, all control animals continued to reach the stimulus discrimination criterion, but only five of the eight cocaine monkeys met the stimulus discrimination criterion. To increase incentive, we increased the reward contrast between stimuli by changing the reward values to $0.12,0.03$, and $0.001 \mathrm{ml} / \mathrm{kg}$ for the high, medium, and low reward values (post-administration high contrast). All animals subsequently met the discrimination criterion, and reversal performance could then be evaluated in seven of the eight animals (there were insufficient remaining trials in one animal). The high reward contrast was used for the subsequent duration of self-administration (nine sessions, over 4-5 months).

\section{Stimulus discrimination/reversal task analysis}

Associative learning was evaluated in two ways. To use a comparable analysis as used for the reversal task, the primary approach was comparison of accuracy over the first 15 trials of the discrimination component, binning every three trials to reduce noise inherent in averaging binary data. We limited our analysis to the first 15 trials presented for each stimulus set to focus on the subject's adaptation to a new stimulus set or the reversal of the reward contingencies. To analyze discrimination performance using the stimulus discrimination/reversal low reward contrast task (for which baseline and post self-administration performance was available), a three-way repeatedmeasures ANOVA was used to make a between-groups comparison of stimulus discrimination performance with bin number and exposure period (baseline vs post self-administration) being the repeated factors. For stimulus discrimination/reversal high reward contrast, only a between-groups comparison of performance in the post self-administration period was possible. A two-way repeated-measures ANOVA was used with bins being the repeated factor. An additional approach was to calculate trials to criterion to assess how well animals were able to sustain high levels of accuracy needed to reach criterion performance on the stimulus discrimination blocks ( 27 of 30 trials correct). If an animal did not meet the stimulus discrimination criteria, a conservative score of 200 trials (the session limit) was assigned. A two-way repeated-measures analysis was used with period (pre-administration low contrast, postadministration low contrast, and post-administration high contrast) being the repeated factor. We followed this with a Holm-Sidak-corrected multiple comparison procedure to evaluate the effect of period within each group.

The measure of cognitive control/flexibility was performance over the first 15 trials after reversal of the reward contingencies, also binned three trials at a time. A two-way repeated-measures ANOVA was used with bins (during the post-administration high reward contrast period) being the repeated factor. It was not possible to compare trials to criterion on the reversal blocks, because the 200 trial sessions always began with a discrimination block, and by the time the stimulus discrimination criterion was reached, thereby initiating a reversal block, there were only a limited number of trials remaining.

\section{Delayed match-to-sample task}

At the start of each trial (total of 160 trials per session), a sample stimulus (an image randomly selected from a 400 image pool) would appear on the touch screen. Pressing the sample stimulus accurately and holding it for $1 \mathrm{~s}$ led to its offset. After a specified delay interval (randomly selected from $0,10,20$, or $40 \mathrm{~s}$ ), the sample and a novel stimulus (randomly selected from the image pool) appeared, randomly assigned to the left and right side of the screen. Choosing the sample stimulus within $10 \mathrm{~s}$ after the presentation of the two stimuli led to a water reward $(0.075$ $\mathrm{ml} / \mathrm{kg}$ ). No reward was delivered for choosing the wrong stimulus or pressing the area outside of the choice stimuli. The intertrial intervals for a correct response and incorrect response were 2 and $7 \mathrm{~s}$, respectively. Baseline performance was determined from 10 daily sessions before initiating self-administration, after which animals performed the task once every 2 weeks to assess visual working memory.

Delayed match-to-sample task analysis. Accuracy was evaluated only using trials in which one of the two stimuli was chosen. Thus, any trials with a non-choice error (omission or touching the screen outside of a stimulus) did not contribute to the measure of choice accuracy. Only sessions in which animals completed at least half of the 160 trials and met a side bias criterion (responses on each side were $\geq 25 \%$ and $\leq 75 \%$ of the trials completed) were included in the analysis. In the cocaine group, 33 of the 152 possible sessions were omitted, and, in the control group, 16 of the 114 possible sessions were removed. Data were analyzed over 19 sessions during the self-administration period. Baseline data were averaged across the 10 sessions before the start of self-administration. The measures used for group matching were accuracy at the $40 \mathrm{~s}$ delay and the area under the curve of accuracy across all delays.

A three-way repeated-measures ANOVA was used to perform a betweengroup comparison of accuracy, with delay interval and exposure period (baseline vs post self-administration) being the repeated factors. To follow up any interaction observed in the three-way repeated-measures ANOVA, a two-way repeated-measures ANOVA was used for a within-group comparison, with period (baseline and post self-administration periods) and delay interval being the repeated measures.

\section{Progressive ratio assessment of water reward efficacy}

A progressive ratio procedure was used for a weekly assessment of efficacy of the water reward used to motivate task performance. Animals touched a stimulus on the screen once for the first water reinforcement $(1.0 \mathrm{ml} / \mathrm{kg})$. The number of responses required for each subsequent water reinforcement doubled, until the animal failed to complete the response requirement within $15 \mathrm{~min}$ or the animal failed to touch the screen for $10 \mathrm{~min}$.

Progressive ratio analysis. The measure of water reward efficacy was the breakpoint, defined as the sequence number of the last reinforcement received (also the power of two equaling the number of responses required) before the session was terminated because responding ceased. Progressive ratio data were collected throughout the entire selfadministration period ( 30 weekly sessions), and the baseline was collected 1 week before the start of self-administration.

\section{Results}

Baseline performance matching of groups and comparability of daily versus intermittent assessments

Baseline performance of the control and cocaine groups on the stimulus discrimination/reversal-low reward contrast and delayed match-to-sample tasks are shown in Figure 2. There were no differences between the groups during the baseline period. There was also not a noticeable drop off in performance on any of the cognitive tasks as a result of going from the daily assessment used to establish baseline performance to assessments once every 2 weeks. This is demonstrated in the comparison of the control group baseline performance and post-administration performance for the stimulus discrimination/reversal task shown in Figure 3 and the delayed matchto-sample task shown in Figure $8 b$. 

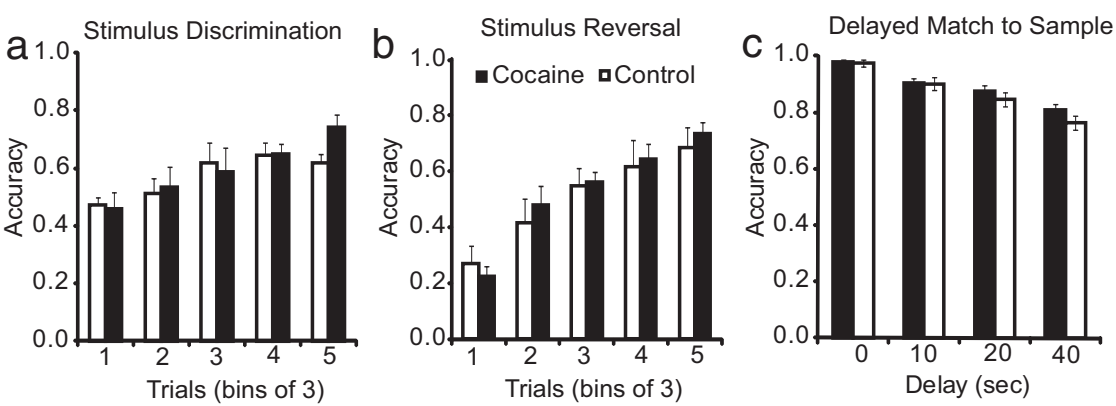

Figure 2. Performance matching of groups before cocaine exposure. Baseline performance on stimulus discrimination (a), reversal $(\boldsymbol{b})$, and delayed match-to-sample $(\boldsymbol{c})$ task is shown. There was no significant difference in performance between groups on any task before initiating self-administration.

\section{Stimulus discrimination performance}

During the first 4-5 months of self-administration, there was no significant effect on accuracy by group or exposure period (baseline, post-administration low reward contrast). In addition, there was no significant group $\times$ exposure period $\times$ trial bin interaction. Associative learning is clearly occurring over those 15 trials, given the main effect of bin number $\left(F_{(4,9)}=28.9, p<0.0001\right)$. Figure 4 shows the comparison between groups in the post self-administration low reward contrast period. Two-way repeated measures on just this exposure period also shows a main effect of trial bin number $\left(F_{(4,9)}=8.85, p<0.001\right)$ without a group $\times$ bin number interaction $(p=0.53)$. However, despite this indication of equivalent associative learning, only five of the eight cocaine monkeys were able to meet the stimulus discrimination criterion of 27 of 30 correct trials. Thus, there was an apparent difficulty in the cocaine group to sustain high levels of accuracy across 30 consecutive trials used for criterion evaluation. This is reflected in a marginal interaction on trials to criterion between group and period (pre-administration low contrast, post-administration low contrast, post-administration high contrast) (group $\times$ period, $\left.F_{(1,24)}=3.283, p=0.055\right)$ (Fig. $\left.5 a\right)$. In the control group, there was no significant difference in trials to criterion between any of the periods. However, it took the cocaine group more trials to reach criterion during the post-administration low contrast compared with the baseline period $\left(t_{(1,7)}=2.61, p=\right.$ $0.015)$. Thus, while maintaining the low reward contrast, there is not an effect of self-administration on trials to criterion in the control group, but there is an impairment in the cocaine group. Increasing the reward contrast significantly decreased trials to criterion in the cocaine group (post-administration low contrast vs post-administration high contrast, $\left.t_{(1,7)}=2.70, p=0.012\right)$. However, increasing reward contrast in the control group had no effect on trials to criterion.

To explore the time course of the increase in trials to criterion, we evaluated performance in three session epochs. Although we lacked the statistical power $(<0.1)$ to confirm a group $\times$ block interaction, the time course of the change in trials to criterion is shown in Figure $5 b$. That change is significantly different between groups when evaluated over the entire post-administration low contrast period (Fig. 5a).

\section{Stimulus reversal performance}

Because not all cocaine animals were meeting discrimination criterion during the post-administration low contrast period, reversal performance could not be evaluated, necessitating the increase in reward contrast between stimuli. All animals subsequently reached criterion on the discrimination component of the stimulus discrimination/reversal task [although one of the eight never reached criterion with enough (15) trials remaining in the fixed
200 limit to allow evaluation of reversal performance]. In contrast to the lack of difference in discrimination accuracy between groups (Fig. 6), accuracy on the reversal learning component during the first 15 trials after the reversal was significantly poorer in the cocaine group, with a twoway repeated-measures ANOVA indicating a main effect of group $\left(F_{(1,11)}=5.13\right.$, $p=0.045)$ and a group $\times$ bin interaction $\left(F_{(1,4)}=3.49, p=0.015\right)$ (Fig. 7).

It is possible that less frequent encounters with reversals contributed to the deficits on reversal performance in the cocaine group. However, a two-way repeated-measures ANOVA of reversals encountered per session did not reveal a group $\times$ period interaction $\left(F_{(1,11)}=2.98, p=0.072\right)$ or a significant main effect. There was also not a significant difference between the two groups in the post-administration high contrast block (independent $t$ test, $\left.t_{(1,11)}=1.08, p=0.30\right)$. Thus, reversal impairments in the cocaine group seen during the post self-administration high contrast condition are not accompanied by less frequent encounters of reversals. Table 2 lists the number of reversals encountered by each group in the different periods, excluding the cocaine animal for which reversal accuracy was not available.

\section{The effects of cocaine self-administration on visual working memory}

A three-way repeated-measures ANOVA of delayed match-tosample accuracy revealed a delay $\times$ period $\times$ group interaction $\left(F_{(3,36)}=8.88, p<0.001\right)$. A period $\times$ group interaction $\left(F_{(1,12)}=\right.$ $5.98, p=0.031)$ and delay $\times$ period interaction $\left(F_{(3,12)}=3.61\right.$, $p=0.022)$ were also revealed. A follow-up two-way repeatedmeasures ANOVA indicated that, in the cocaine group, there was a main effect of period $\left(F_{(1,7)}=8.62, p=0.02\right)$ and a significant interaction between period and delay $\left(F_{(3,36)}=4.61, p=0.012\right)$, with accuracy decreased after cocaine self-administration compared with the baseline period (Fig. $8 a$ ). The delay dependency of the decreased accuracy is consistent with specific impairments in working memory after cocaine self-administration. The control group did not show a significant difference in accuracy (Fig. 8b) when comparing baseline performance with that during the selfadministration period $\left(F_{(1,5)}=0.56, p=0.49\right)$ nor an interaction. To explore the time course of the decrease in working memory, we compared accuracy at the 40 s delay, averaged over four session epochs to baseline. A one-way repeated-measures ANOVA revealed that the first post-administration epoch was significantly different from baseline. Increased variability associated with fewer sessions obscured differences from baseline in the later epochs. The time course for this metric is shown for both cocaine and control groups in Figure $8 c$.

\section{Comparison of impairments across cognitive domains}

The extent to which impairments in one cognitive domain extend to another can help to address the possibility of a common underlying substrate or mechanism. To examine this, we evaluated the regression of changes in working memory performance before and after self-administration with those of reversal performance. The metrics used were accuracy at the $40 \mathrm{~s}$ delay interval and the slope of reversal accuracy across the first five (three trial) bins after stimulus reversal. There was a marginally significant regression $\left(F_{(1,6)}=5.76, p=\right.$ 0.062 ) (Fig. 9), consistent with the possibility of a common mechanism of impairment on the two tasks. 

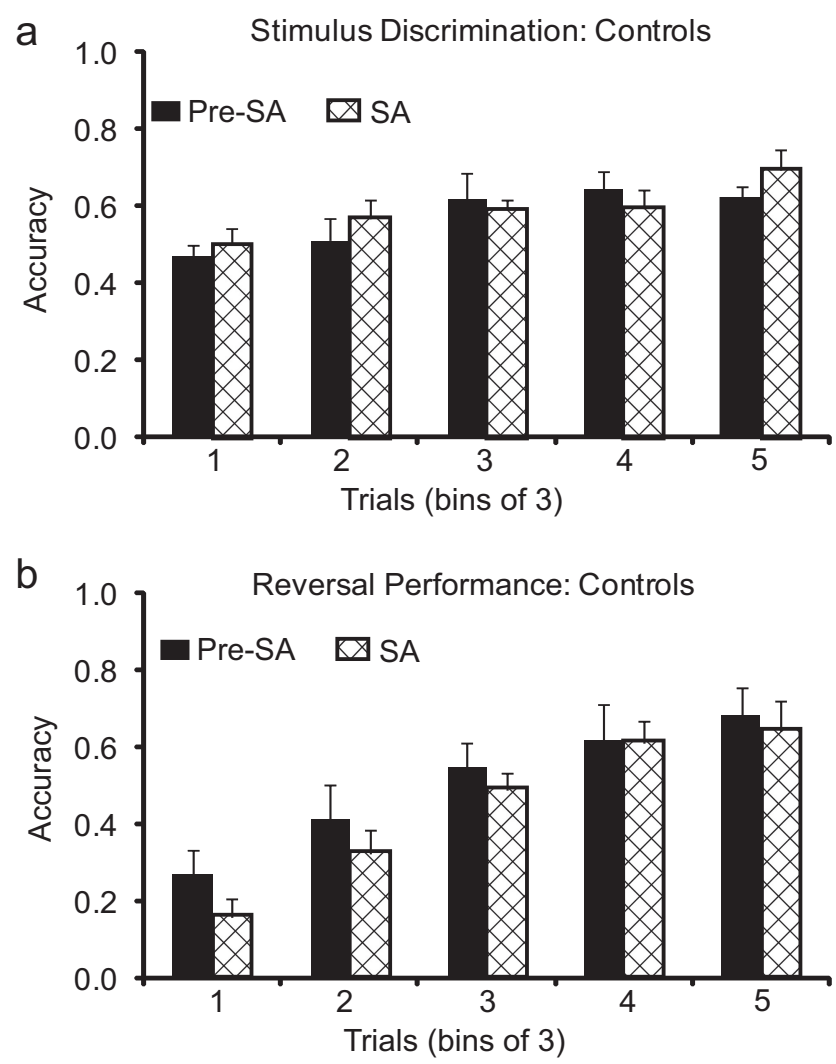

Figure 3. Validity of intermittent cognitive assessments. Comparison of baseline performance based on 10 consecutive daily sessions with subsequent assessments conducted at 2 week intervals, control group only. By two-way repeated-measures ANOVA, there was no effect of pre-administration versus post-administration for stimulus discrimination $\left(F_{(1,5)}=0.35\right.$, $p=0.58)$ or reversal performance $\left(F_{(1,5)}=1.11, p=0.34\right)$. SA, Self-administration.

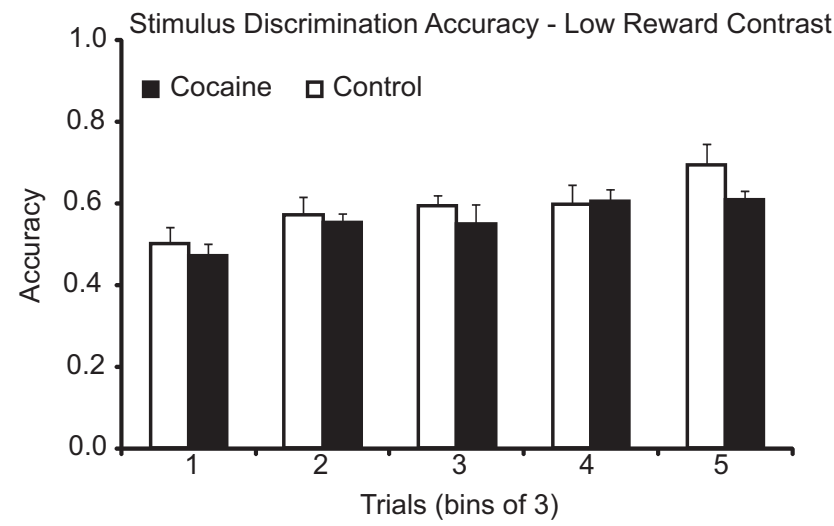

Figure 4. Between-groups comparison of stimulus discrimination performance on the low reward contrast task. Over the first 15 trials of nine sessions conducted over $\sim 5$ months of self administration, there was no significant between group difference in discrimination accuracy.

\section{Impact of cocaine self-administration on water reward efficacy}

Water reward efficacy (breakpoint on a progressive ratio task) was assessed weekly in both groups throughout the entire selfadministration period (30 sessions). As can be seen in Figure 10, there was no difference between groups in breakpoint over the self-administration period.

\section{Discussion}

The nature and extent of cognitive deficits associated with cocaine use have been widely studied. However, whether they rep-
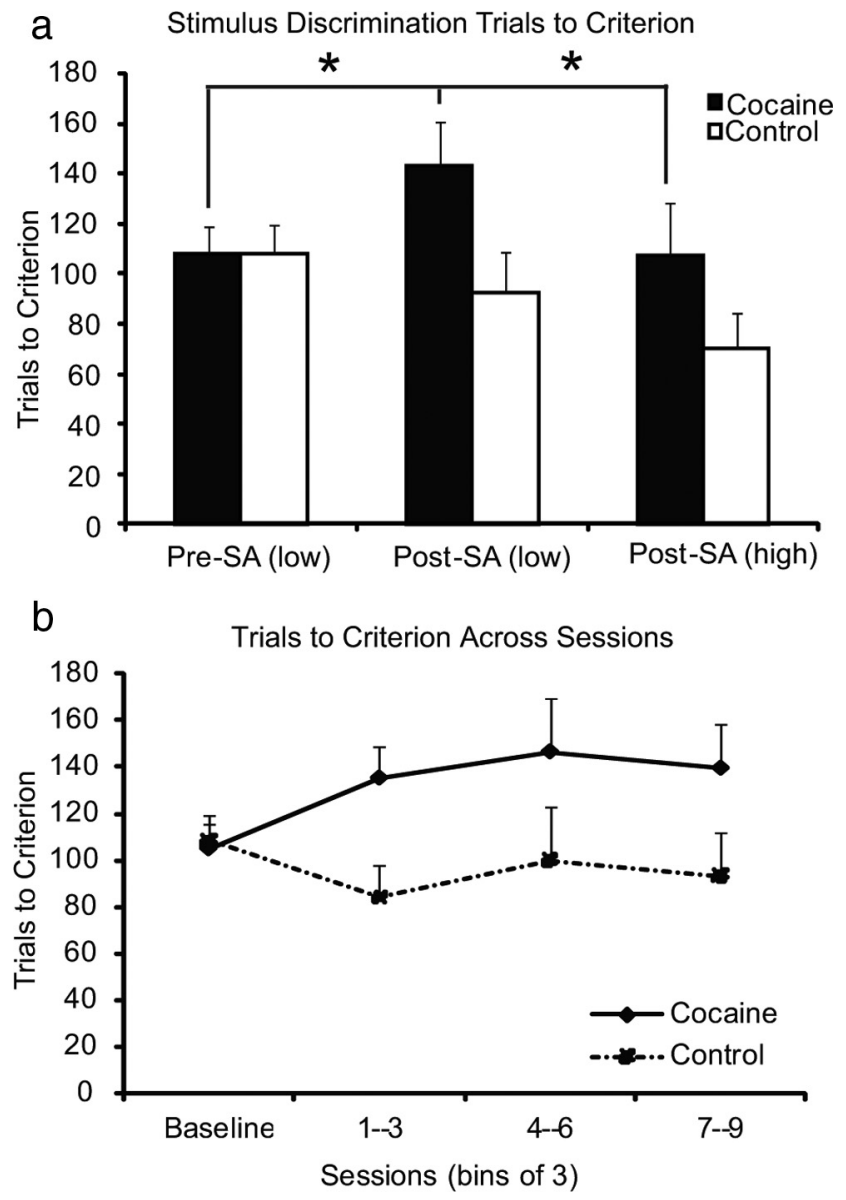

Figure 5. Stimulus discrimination trials to criterion. $\boldsymbol{a}$, Between-groups comparison of trials to criterion on the stimulus discrimination task across conditions. Pre-SA (low), Baseline before self-administration, low reward contrast; Post-SA (low), sessions during self-administration over the first 4-5 months, low reward contrast; Post-SA (high), sessions during selfadministration months 5-9, high reward contrast. Group $\times$ period interaction: $F_{(1,24)}=3.28$, $p=0.055$. $\boldsymbol{b}$, Stimulus discrimination performance across all sessions of the stimulus discrimination low bias task. Sessions were binned by three. ${ }^{*} p<0.05$.

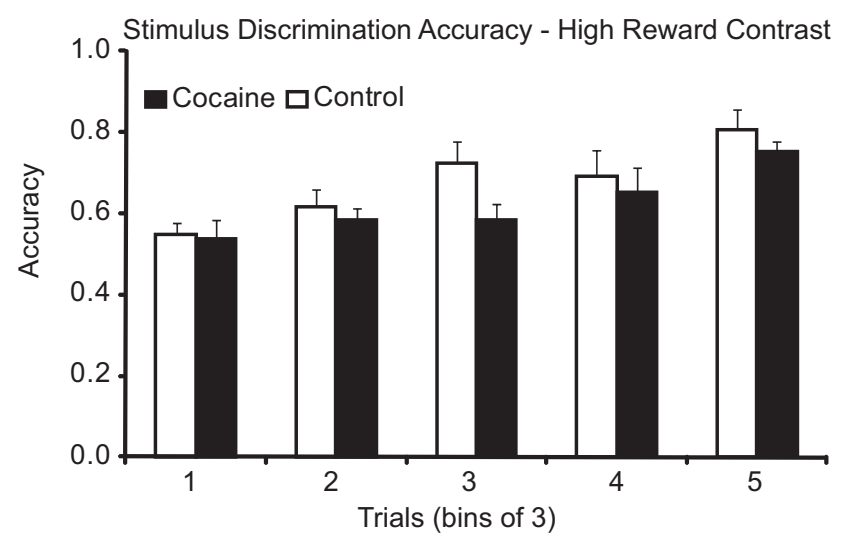

Figure 6. Between-group comparison of stimulus discrimination performance on the high reward contrast stimulus discrimination/reversal task, months $5-9$ post self-administration. There was no significant difference between groups on discrimination accuracy $\left(F_{(1,4)}=1.70, p=0.165\right)$.

resent predisposing traits or consequences of drug use is difficult to assess in clinical investigations. We demonstrate by longitudinal assessment of cognitive function across multiple domains in a nonhuman primate model that cocaine self-administration 


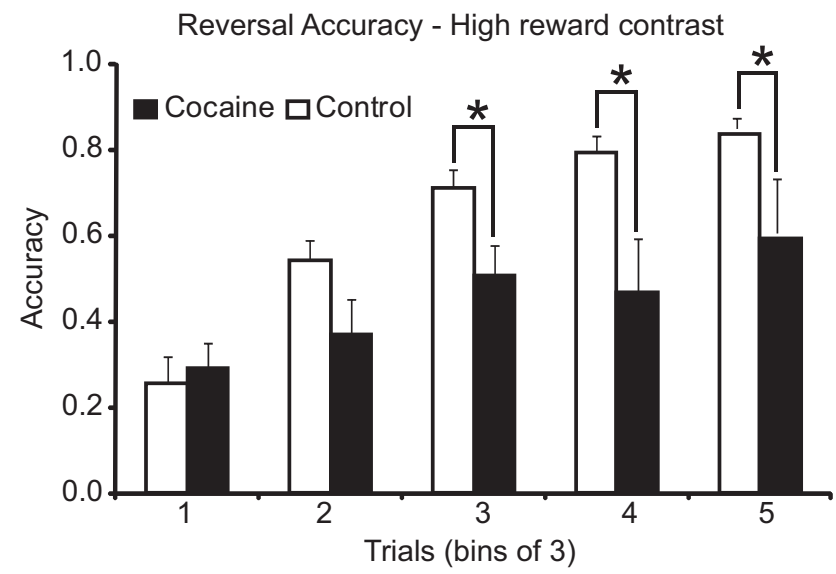

Figure 7. Between-group comparison of reversal performance on the high reward contrast stimulus discrimination/reversal task during months $5-9$ of self-administration. The cocaine group shows a decrease in accuracy compared with controls. Two-way repeated-measures ANOVA shows a main effect of group $\left(F_{(1,11)}=5.13, p=0.045\right)$ and a group $\times$ bin interaction $\left(F_{(1,4)}=3.49, p=0.015\right) .{ }^{*} p<0.05$.

Table 2. Number of reversals encountered per session

\begin{tabular}{lll}
\hline Period & Control & Cocaine \\
\hline Baseline & $1.00 \pm 0.10$ & $1.14 \pm 0.16$ \\
Post-administration low contrast & $1.22 \pm 0.17$ & $0.67 \pm 0.20$ \\
Post-administration high contrast & $1.51 \pm 0.26$ & $1.12 \pm 0.27$ \\
\hline
\end{tabular}

Number of times per session each group encountered a reversal over the three blocks (baseline, post-administration low contrast, post-administration high contrast). Control, $n=6$; cocaine, $n=7$.

causes strong deficits in cognitive control/flexibility. Less pronounced deficits in visual working memory (delayed match-tosample) and the ability to maintain a stimulus discrimination were also observed.

A stimulus discrimination/reversal task was used to assess associative learning and cognitive control/flexibility. During the first 4-5 months of self-administration, we did not observe a significant difference between the groups on stimulus discrimination accuracy, evaluated over the first 15 trials after presentation of a new set of stimuli. However, unlike all animals from both groups during baseline performance and all control animals during self-administration, only five of the eight cocaine animals were able to meet the stimulus discrimination criteria of 27 of 30 consecutively correct trials within a 200 trial session. This suggests intact associative learning but difficulty in maintaining the high level of accuracy needed to reach criterion performance.

The improved discrimination performance after increasing the reward contrast between stimuli permitted a comparison of cognitive control/flexibility (reversal performance) between groups. The cocaine group was less able to adapt their responding to the reversal of the reward contingencies, consistent with the clinical literature indicating that chronic cocaine users show impaired reversal performance, whereas the acquisition of an initial stimulus-reward association was not impaired (Fillmore and Rush, 2006; Ersche et al., 2008). These results also extend previous findings with noncontingent cocaine exposure in vervet monkeys (Jentsch et al., 2002) and rodents (Schoenbaum et al., 2004). Although a reduced frequency of encountering reversals during the post-administration low contrast period could have contributed to the impaired reversal performance, the task had been well learned before initiating self-administration, and there was no difference in frequency of reversals during the postadministration high contrast period. Although recent reports
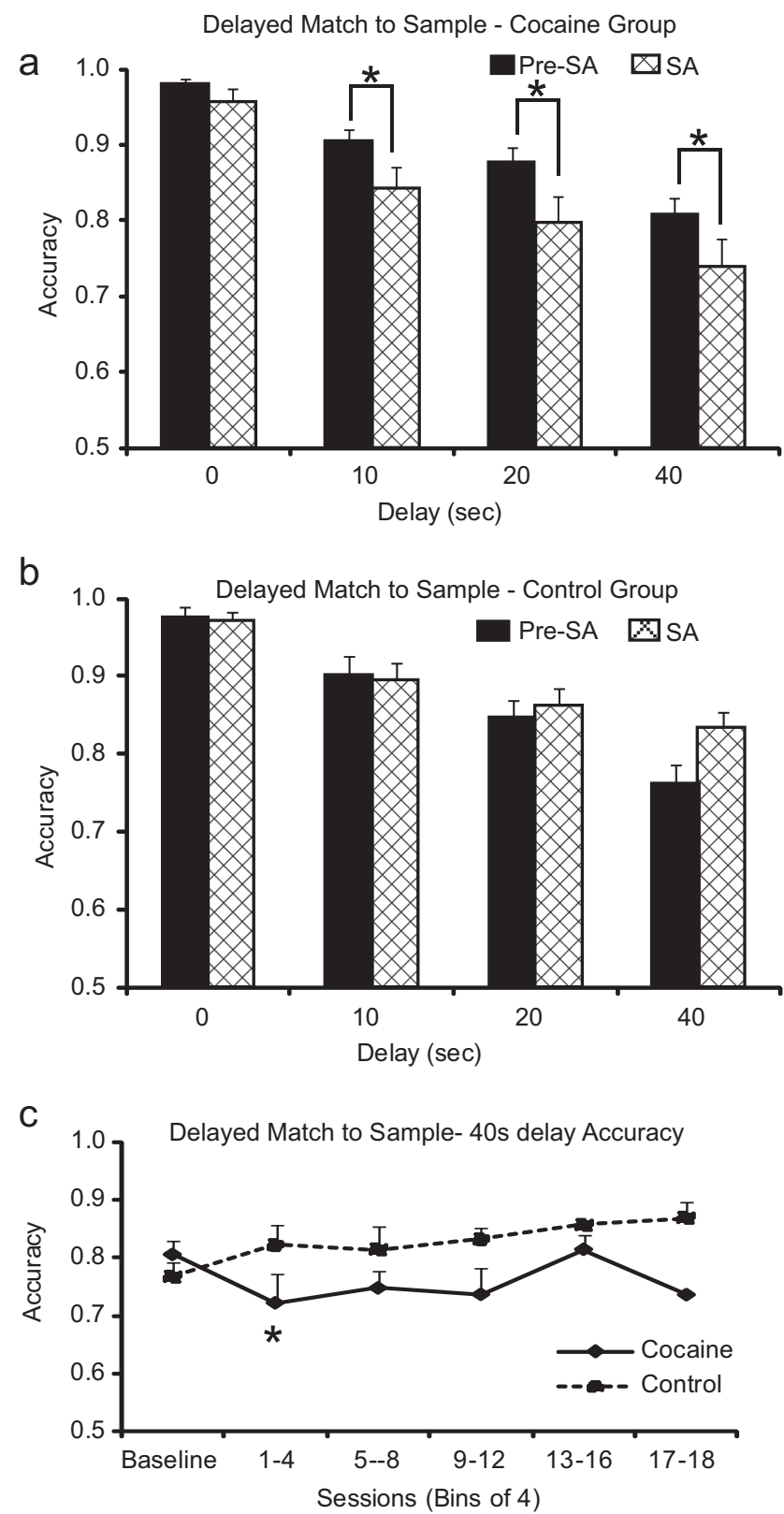

Figure 8. Within-group comparison of delayed match-to-sample performance. $\boldsymbol{a}$, In the cocaine group, there was a main effect of period $\left(F_{(1,7)}=8.62, p=0.02\right)$ and a significant interaction between period and delay $\left(F_{(3,36)}=4.61, p=0.012\right)$, with accuracy decreased after cocaine self-administration (SA) compared with the baseline period. $\boldsymbol{b}$, The control group did not show a significant difference in accuracy when comparing baseline with the selfadministration period. $c$, Time course of changes in accuracy at the $40 \mathrm{~s}$ delay of the delayed match-to-sample task across all sessions. Sessions were binned by four. After one-way repeated-measures ANOVA, post hoc analysis (Holm-Sidak) indicated the cocaine group had impaired working memory during sessions $1-4$ after self-administration compared with baseline. ${ }^{*} p<0.05$.

indicate that damage to medial striatum can impair reversal performance (Clarke et al., 2008), such effects are most frequently associated with orbitofrontal cortex dysfunction (Butter, 1969; Hornak et al., 2004; Izquierdo et al., 2004). This region shows structural (Franklin et al., 2002) and functional (Volkow et al., 1993; Grant et al., 2000; London et al., 2000) abnormalities in drug using populations. Given its role in inhibitory control and decision-making, it plays a central role in heuristic conceptualizations of cortical systems implicated in addiction and other syndromes of impaired consumptive inhibitory 
Comparison of Working Memory and Reversal Deficits

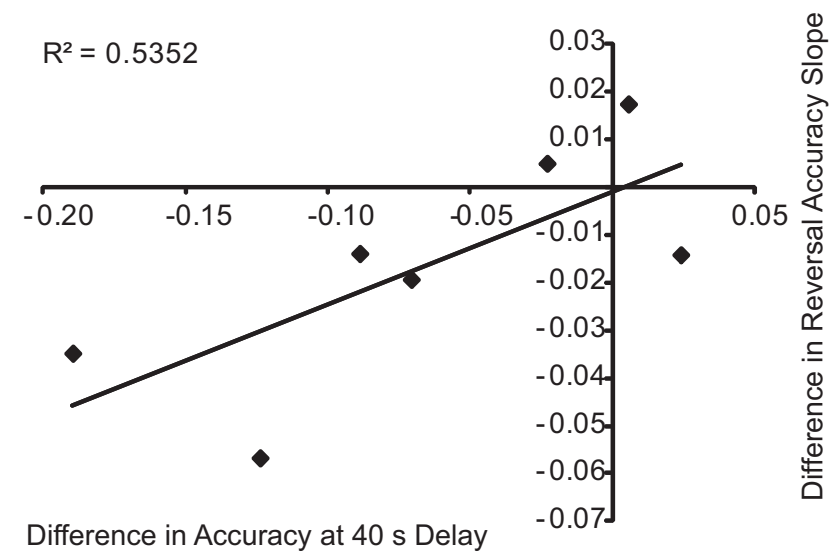

Figure 9. Regression of memory deficits with reversal deficits in the cocaine group. $x$-Axis is the difference in accuracy at the $40 \mathrm{~s}$ delay of the delayed match-to-sample task. $y$-Axis is the difference in the reversal accuracy slope across the first 15 trials of each session. There was a marginally significant correlation between change in working memory and reversal performance. $R^{2}=0.535, p=0.062$.

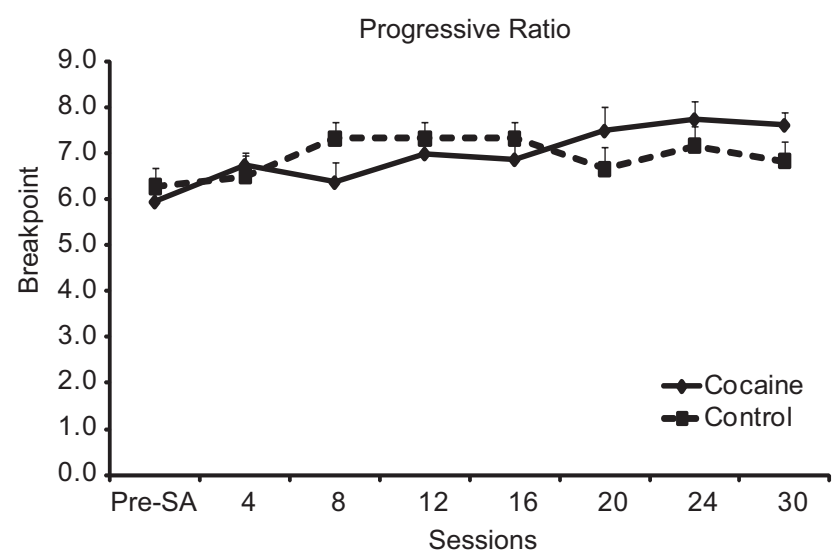

Figure 10. Between-group comparison of water reward efficacy, measured by breakpoint on a progressive ratio schedule of self-administration (SA). There was no difference between groups either before or after self-administration.

control (Bechara, 2005; Schoenbaum et al., 2006; Schoenbaum and Shaham, 2008; Volkow et al., 2008).

There have been inconsistencies in the literature in regard to whether cocaine exposure affects working memory. Some clinical studies (Hoff et al., 1996; Bechara and Martin, 2004; Kübler et al., 2005) and a rodent self-administration study (George et al., 2008) have shown working memory deficits in cocaine using populations. In other cases, no deficits have been reported (Bolla et al., 1999; Pace-Schott et al., 2008). That inconsistency may be a reflection of a modest effect size. We were able to match performance very carefully between our groups before any exposure. That, combined with a longitudinal approach, enabled us to observe what appears to be a clear, although relatively modest, working memory impairment that was apparent soon after initiating exposure. Although visual working memory is usually associated with ventrolateral prefrontal cortex (Wilson et al., 1993), orbitofrontal contributions are also apparent (Otto and Eichenbaum, 1992; Wallis, 2007), consistent with the marginally significant relationship we observed between working memory and reversal impairments.

It appears that deficits emerge relatively quickly after initiation of self-administration. All assessments were made $3 \mathrm{~d}$ after last exposure; thus, a "short-term withdrawal" effect is possible. Withdrawal is a difficult concept to address with cocaine use. Preliminary results indicate no difference in total sleep and average sleep epoch duration between groups over the weekend before testing (Gomez et al., 2010). Thus, dysregulation of the pharmacological targets of cocaine is more likely than a generalized fatigue or sleep deprivation.

It has been noted that performance on a wide range of cognitive tasks is often inextricably linked to attention and reward (Maunsell, 2004; Sarter et al., 2006; Kennerley and Wallis, 2009). We believe the progressive ratio evaluation of water reward efficacy indicates no difference between our groups in motivation. However, increasing the reward contrast between stimuli on the discrimination task improved performance in the cocaine but not the control group. Thus, it appears that an interaction between reward incentive and task difficulty (Maunsell, 2004) distinguishes between our groups. For very simple tasks, such as the progressive ratio task in which only repetitive touching to a nonmoving stimulus is needed, the incentive of water reward is unchanged. However, for a more difficult task, such as the stimulus discrimination or perhaps the working memory task, greater rewards are needed to support the focused attention required by the task (Maunsell, 2004). Given the importance of dopamine in signaling rewards (Schultz, 2007) and the clear dysregulation by cocaine of dopamine systems (Weiss et al., 1992; Letchworth et al., 2001; Nader et al., 2002, 2006) and reward circuitry in general (Kalivas and Volkow, 2005), it is also possible that reward incentives are communicated across systems less effectively, requiring a greater contrast between different rewards to effectively engage cognitive networks.

Attentional impairments are possible in the cocaine-exposed animals, given their difficulty in maintaining high levels of accuracy on the discrimination task needed to achieve criterion. We have also presented preliminary results of increased intraindividual variability in response times in the cocaine animals (Olsen et al., 2009), an observation consistent with greater attentional lapses (Castellanos et al., 2005; Olsen et al., 2009), and increased distractibility in them as well (Porter et al., 2010). Thus, to the extent that working memory and attention are overlapping, increased distractibility or attentional lapses could have contributed to errors on the delayed match-to-sample task (Duncan, 2001). A common substrate, such as impaired attention, as a contributor to the general pattern of deficits is also suggested by the trend toward a correlation of impairments in working memory and reversal performance across individuals.

In contrast to the current findings in which a well-learned discrimination task did not reveal impairments in accuracy over the first 15 trials after cocaine self-administration, we reported previously (Liu et al., 2008) that initial acquisition of a stimulus discrimination task and a spatial working memory task was significantly impaired in a group of monkeys with a history of selfadministration. After extensive training, animals that showed impaired stimulus discrimination task acquisition eventually became equally adept as the control group (our unpublished results). This pattern of acquisition impairment is similar to the effects of orbitofrontal lesions (Otto and Eichenbaum, 1992). We believe the more pronounced discrimination impairment seen previously (Liu et al., 2008) reflects an inability to focus on the relevant structure of a new task to be acquired in the animals with a history of drug exposure. In contrast, if a learning set has already been established by experience with the task (Harlow, 1949), performance is much less impaired by experimental manipulations, as the present study indicates. 
The contrast between effects of cocaine on novel task acquisition versus performance of a familiar one, and the somewhat generalized effects observed, is consistent with the adaptive coding model of prefrontal cortex function proposed by Duncan (2001). That model posits a less compartmentalized and regionally specific distribution of function than often presumed, evidenced by the high level of adaptability of broad areas of prefrontal cortex to accommodate specific task contingencies. It is proposed that part of the function by which the prefrontal cortex mediates acquisition of task contingencies is also by minimizing distractibility, or increasing focused attention. To quote Duncan, "In this model, working memory, selective attention and control are simply three different perspectives on the same underlying processing function" (Duncan, 2001). We also feel that Sarter's description of "attentional effort" (Sarter et al., 2006) needed for task performance is an intuitively appealing description of the impairment in the cocaine-exposed animals. Thus, the overall nature of the impairments appears to be a mixture of highly selective effects on orbitofrontal cortex associated reversal performance along with a more generalized impairment of prefrontal-dependent working memory/attentional focus.

These results represent a unique study in which a broad range of cognitive domains were studied longitudinally in nonhuman primates to determine the effects of chronic cocaine selfadministration. The results strongly suggest that, in addition to the substantial literature indicating the contribution of inherent differences between individuals for risk of addiction (DerocheGamonet et al., 2004; Tarter et al., 2007; Belin et al., 2008), cocaine use by itself causes cognitive deficits. Understanding the neurobiological basis of these deficits may help in the development of therapeutic approaches to address them, potentially increasing the likelihood of abstinence based on the links between cognitive performance and treatment outcome.

\section{References}

Aharonovich E, Hasin DS, Brooks AC, Liu X, Bisaga A, Nunes EV (2006) Cognitive deficits predict low treatment retention in cocaine dependent patients. Drug Alcohol Depend 81:313-322.

Bechara A (2005) Decision making, impulse control and loss of willpower to resist drugs: a neurocognitive perspective. Nat Neurosci 8:1458-1463.

Bechara A, Martin EM (2004) Impaired decision making related to working memory deficits in individuals with substance addictions. Neuropsychology 18:152-162.

Belin D, Mar AC, Dalley JW, Robbins TW, Everitt BJ (2008) High impulsivity predicts the switch to compulsive cocaine-taking. Science 320:1352-1355.

Bolla KI, Rothman R, Cadet JL (1999) Dose-related neurobehavioral effects of chronic cocaine use. J Neuropsychiatry Clin Neurosci 11:361-369.

Butter CM (1969) Perseveration in distinction and in discrimination reversal tasks following selective frontal ablations in Macaca mulatta. Physiol Behav 4:163-171.

Castellanos FX, Sonuga-Barke EJ, Scheres A, Di Martino A, Hyde C, Walters JR (2005) Varieties of attention-deficit/hyperactivity disorder-related intra-individual variability. Biol Psychiatry 57:1416-1423.

Clarke HF, Robbins TW, Roberts AC (2008) Lesions of the medial striatum in monkeys produce perseverative impairments during reversal learning similar to those produced by lesions of the orbitofrontal cortex. J Neurosci 28:10972-10982.

Deroche-Gamonet V, Belin D, Piazza PV (2004) Evidence for addiction-like behavior in the rat. Science 305:1014-1017.

Dias R, Robbins TW, Roberts AC (1996) Dissociation in prefrontal cortex of affective and attentional shifts. Nature 380:69-72.

Duncan J (2001) An adaptive coding model of neural function in prefrontal cortex. Nat Rev Neurosci 2:820-829.

Elliott R, Dolan RJ (1999) Differential neural responses during performance of matching and nonmatching to sample tasks at two delay intervals. J Neurosci 19:5066-5073.
Ersche KD, Roiser JP, Robbins TW, Sahakian BJ (2008) Chronic cocaine but not chronic amphetamine use is associated with perseverative responding in humans. Psychopharmacology (Berl) 197:421-431.

Fellows LK, Farah MJ (2003) Ventromedial frontal cortex mediates affective shifting in humans: evidence from a reversal learning paradigm. Brain 126:1830-1837.

Fillmore MT, Rush CR (2006) Polydrug abusers display impaired discrimination-reversal learning in a model of behavioural control. J Psychopharmacol 20:24-32.

Franklin TR, Acton PD, Maldjian JA, Gray JD, Croft JR, Dackis CA, O'Brien CP, Childress AR (2002) Decreased gray matter concentration in the insular, orbitofrontal, cingulate, and temporal cortices of cocaine patients. Biol Psychiatry 51:134-142.

Garavan H, Hester R (2007) The role of cognitive control in cocaine dependence. Neuropsychol Rev 17:337-345.

George O, Mandyam CD, Wee S, Koob GF (2008) Extended access to cocaine self-administration produces long-lasting prefrontal cortexdependent working memory impairments. Neuropsychopharmacology 33:2474-2482.

Gomez G, Jedema HP, Gurnsey K, Bradberry CW (2010) The effect of cocaine self administration on sleep in rhesus monkeys. Society Neurosci Abstr 36:506.20.

Grant S, Contoreggi C, London ED (2000) Drug abusers show impaired performance in a laboratory test of decision making. Neuropsychologia 38:1180-1187.

Harlow HF (1949) The formation of learning sets. Psychol Rev 56:51-65.

Hoff AL, Riordan H, Morris L, Cestaro V, Wieneke M, Alpert R, Wang GJ, Volkow N (1996) Effects of crack cocaine on neurocognitive function. Psychiatry Res 60:167-176.

Hornak J, O'Doherty J, Bramham J, Rolls ET, Morris RG, Bullock PR, Polkey CE (2004) Reward-related reversal learning after surgical excisions in orbito-frontal or dorsolateral prefrontal cortex in humans. J Cogn Neurosci 16:463-478.

Izquierdo A, Suda RK, Murray EA (2004) Bilateral orbital prefrontal cortex lesions in rhesus monkeys disrupt choices guided by both reward value and reward contingency. J Neurosci 24:7540-7548.

Jentsch JD, Olausson P, De La Garza R 2nd, Taylor JR (2002) Impairments of reversal learning and response perseveration after repeated, intermittent cocaine administrations to monkeys. Neuropsychopharmacology 26:183-190.

Kalivas PW, Volkow ND (2005) The neural basis of addiction: a pathology of motivation and choice. Am J Psychiatry 162:1403-1413.

Kennerley SW, Wallis JD (2009) Reward-dependent modulation of working memory in lateral prefrontal cortex. J Neurosci 29:3259-3270.

Kübler A, Murphy K, Garavan H (2005) Cocaine dependence and attention switching within and between verbal and visuospatial working memory. Eur J Neurosci 21:1984-1992.

Letchworth SR, Nader MA, Smith HR, Friedman DP, Porrino LJ (2001) Progression of changes in dopamine transporter binding site density as a result of cocaine self-administration in rhesus monkeys. J Neurosci 21:2799-2807.

Liu S, Heitz RP, Sampson AR, Zhang W, Bradberry CW (2008) Evidence of temporal cortical dysfunction in rhesus monkeys following chronic cocaine self-administration. Cereb Cortex 18:2109-2116.

London ED, Ernst M, Grant S, Bonson K, Weinstein A (2000) Orbitofrontal cortex and human drug abuse: functional imaging. Cereb Cortex 10:334-342.

Maunsell JH (2004) Neuronal representations of cognitive state: reward or attention? Trends Cogn Sci 8:261-265.

Murray EA, Izquierdo A (2007) Orbitofrontal cortex and amygdala contributions to affect and action in primates. Ann N Y Acad Sci 1121:273-296.

Nader MA, Daunais JB, Moore T, Nader SH, Moore RJ, Smith HR, Friedman DP, Porrino LJ (2002) Effects of cocaine self-administration on striatal dopamine systems in rhesus monkeys: initial and chronic exposure. Neuropsychopharmacology 27:35-46.

Nader MA, Morgan D, Gage HD, Nader SH, Calhoun TL, Buchheimer N, Ehrenkaufer R, Mach RH (2006) PET imaging of dopamine D2 receptors during chronic cocaine self-administration in monkeys. Nat Neurosci 9:1050-1056.

Olsen AS, Porter JN, Dugan BP, Gurnsey K, Jedema HP, Bradberry CW (2009) Cocaine self-administration impairs attention as shown by in- 
creased intra-subject response time variability in rhesus monkeys. Society Neurosci Abstr 35:158.5.

O’Malley S, Adamse M, Heaton RK, Gawin FH (1992) Neuropsychological impairment in chronic cocaine abusers. Am J Drug Alcohol Abuse $18: 131-144$

Otto T, Eichenbaum H (1992) Complementary roles of the orbital prefrontal cortex and the perirhinal-entorhinal cortices in an odor-guided delayed-nonmatching-to-sample task. Behav Neurosci 106:762-775.

Pace-Schott EF, Morgan PT, Malison RT, Hart CL, Edgar C, Walker M, Stickgold R (2008) Cocaine users differ from normals on cognitive tasks which show poorer performance during drug abstinence. Am J Drug Alcohol Abuse 34:109-121.

Patkar AA, Murray HW, Mannelli P, Gottheil E, Weinstein SP, Vergare MJ (2004) Pre-treatment measures of impulsivity, aggression and sensation seeking are associated with treatment outcome for African-American cocaine-dependent patients. J Addict Dis 23:109-122.

Porter JN, Jedema HP, Dugan BP, Gomez G, Gurnsey K, Bradberry CW (2010) Long lasting increases in distractibility in rhesus monkeys following chronic cocaine self-administration. Soc Neurosci Abstr 36:506.19.

Rogers RD, Robbins TW (2001) Investigating the neurocognitive deficits associated with chronic drug misuse. Curr Opin Neurobiol 11:250-257.

Sarter M, Gehring WJ, Kozak R (2006) More attention must be paid: the neurobiology of attentional effort. Brain Res Rev 51:145-160.

Schoenbaum G, Shaham Y (2008) The role of orbitofrontal cortex in drug addiction: a review of preclinical studies. Biol Psychiatry 63:256-262.

Schoenbaum G, Saddoris MP, Ramus SJ, Shaham Y, Setlow B (2004) Cocaine-experienced rats exhibit learning deficits in a task sensitive to orbitofrontal cortex lesions. Eur J Neurosci 19:1997-2002.

Schoenbaum G, Roesch MR, Stalnaker TA (2006) Orbitofrontal cortex, decision-making and drug addiction. Trends Neurosci 29:116-124.
Schultz W (2007) Behavioral dopamine signals. Trends Neurosci 30: 203-210.

Tarter RE, Kirisci L, Feske U, Vanyukov M (2007) Modeling the pathways linking childhood hyperactivity and substance use disorder in young adulthood. Psychol Addict Behav 21:266-271.

Teichner G, Horner MD, Roitzsch JC, Herron J, Thevos A (2002) Substance abuse treatment outcomes for cognitively impaired and intact outpatients. Addict Behav 27:751-763.

Verdejo-Garcia A, Perez-Garcia M (2007) Profile of executive deficits in cocaine and heroin polysubstance users: common and differential effects on separate executive components. Psychopharmacology (Berl) 190:517-530

Volkow ND, Fowler JS, Wang GJ, Hitzemann R, Logan J, Schlyer DJ, Dewey SL, Wolf AP (1993) Decreased dopamine D2 receptor availability is associated with reduced frontal metabolism in cocaine abusers. Synapse 14:169-177.

Volkow ND, Wang GJ, Fowler JS, Telang F (2008) Overlapping neuronal circuits in addiction and obesity: evidence of systems pathology. Philos Trans R Soc Lond B Biol Sci 363:3191-3200.

Wallis JD (2007) Orbitofrontal cortex and its contribution to decisionmaking. Annu Rev Neurosci 30:31-56.

Weiss F, Markou A, Lorang MT, Koob GF (1992) Basal extracellular dopamine levels in the nucleus accumbens are decreased during cocaine withdrawal after unlimited-access self-administration. Brain Res 593:314-318.

Wilson FA, Scalaidhe SP, Goldman-Rakic PS (1993) Dissociation of object and spatial processing domains in primate prefrontal cortex. Science 260:1955-1958.

Wojnicki FH, Bacher JD, Glowa JR (1994) Use of subcutaneous vascular access ports in rhesus monkeys. Lab Anim Sci 44:491-494. 